\title{
Breakdowns on the information highway during inter-hospital patient transfers
}

\author{
William M. Tierney, MD \\ Department of Population Health, Dell Medical School, University of Texas at Austin, Austin, TX, USA.
}

J Gen Intern Med 33(9):1415-6

DOI: $10.1007 / \mathrm{s} 11606-018-4538-\mathrm{Z}$

(c) Society of General Internal Medicine 2018

I was a member of the team of physicians and technicians who developed, deployed, and studied one of the country's first electronic health record (EHR) systems that were implemented in a large vertically integrated safety net health system. 'By 1986, we had largely completed its development: the system had a comprehensive database of all laboratory test results, imaging study results, coded diagnoses from all inpatient and outpatient sites, and free-text reports of all inpatient and outpatient procedures. ${ }^{2}$ A user-friendly data viewer allowed physicians and other clinicians' easy access to patients EHRs from any clinical site. We had also implemented one of the nation's first computer-based systems for writing all inpatient and outpatient orders and notes, one that physicians actually liked, and a widely utilized and intensively studied clinical decision support system.

Our hospital did not have a cardiovascular surgery suite because it was two blocks from the only university hospital in the state. Nor did we have a neonatal intensive care unit because right across the street was the only children's hospital in the state. Yet despite having a state-of-the-art EHR system, when patients were transferred to the university or children's hospital, as often as not no records accompanied them. And when patients arrived back at our public teaching hospital from the university or children's hospital, they frequently arrived without any records describing what had been done and what still needed to be done. Despite our having a stateof-the-art information system and these three hospitals being part of the same academic medical center, inter-hospital communication was dismal.

In response, Clem McDonald, one of the founding godfathers of biomedical informatics, tapped into the various information subsystems in the university and children's hospitals and created the nation's first health information exchange (HIE). ${ }^{6}$ In 1989, he added the Indianapolis VA Medical Center, located three blocks away. Then over the ensuing 5 years, Clem convinced the other four major hospitals in Central Indiana to provide data in real time to form the Indiana Network for Patient Care (INPC) ${ }^{7}$ that today includes more

Published online June 25, 2018 than 11 billion observations for almost 14 million patients, downloaded in real time from 117 of Indiana's approximately 125 hospitals and more than 40,000 physicians and other health care providers.

During this time, I practiced in this safety net health system as a primary care physician, emergency department physician, and hospitalist. I routinely checked the INPC when caring for a new patient or dealing with a new problem in an established patient. Many times access to a recent workup at another facility or noting that a laboratory abnormality was unchanged prevented me from admitting a patient or initiating an unnecessary diagnostic or therapeutic action. I almost always had the information I needed to care for patients transferred to my care.

It all made perfect sense: health care is an information business - we help patients manage their care through the information we gather via histories, physical exams, laboratory, and imaging tests, etc. Hence, the efficiency, effectiveness, and outcomes of care should be improved by having access to an HIE. Yet data supporting this assumption are sadly lacking. Two systematics reviews, ${ }^{9}{ }^{10}$ and one meta-analysis ${ }^{11}$ found that emergency department use and selected costs might be lower among HIE users, but the evidence was weak and inconsistent. Other benefits were not found. A more recent systematic review is encouraging, but results were still mixed. ${ }^{12}$

How could this be? If having information about our patients is good, why have HIEs not proven beneficial? There are several possible reasons. Studying major changes in health systems is hard, and rigorous randomized, controlled trials are usually impossible. And just because a practice, hospital, or health system is participating in an HIE does not mean their clinicians use it. HIEs may not be user-friendly: even a single extra mouse click will reduce the use of digital health tools. The HIEs may be incomplete or otherwise lack the information being sought, further discouraging their use. Technical issues such as authenticating users' identities, difficulties logging onto such systems, slow network speed, and user-hostile interfaces may frustrate time-stressed clinicians.

Yet the digital information era is here to stay in health care, and instead of definitive studies, we must rely on the preponderance of available evidence to assess whether HIEs provide clinical benefit to patients. The current issue of JGIM contains such evidence. ${ }^{13}$ Usher and his colleagues used data from AHRQ's Health Care Utilization Project (HCUP) ${ }^{14}$ to study hospital transfers. Specifically, they assessed how often 
diagnoses changed between transferring and receiving hospitals, whether gaining or losing documented diagnoses was associated with increased mortality, and whether any adverse effects might be mitigated by hospitals' participating in HIEs. They found that discordance in diagnoses after hospital transfer was common ( $73 \%$ of transferred patients gained one or more diagnosis while $47 \%$ lost one or more) and was associated with an $11 \%$ increase in the odds of dying in the receiving hospital. However, when both transferring and receiving hospitals reported participating in an HIE, diagnostic discordance was less and mortality was $12 \%$ lower.

Again, on their face, these results make perfect sense: when information is lost during patient handoffs, care and outcomes suffer. ${ }^{15}$ When both sides of the handoff can share information through an HIE, one would expect fewer bobbles, fewer critical information discontinuities, and better outcomes. Both the clinician and informatician in me want to believe this. Yet association does not mean causation. The HCUP diagnoses come from discharge case abstracts, and hence it is not surprising that clinicians in the receiving hospitals, with longer lengths of stay ( 8.2 vs. 2.8 days in transferring hospitals), recorded more diagnoses (11.4 vs. 8.1) and comorbidities (3.4 vs. 2.5 ). The authors imply that discordance in diagnoses resulted in increased mortality, yet the opposite seems more likely: patients who died were sicker, had more adverse events, and hence more recorded diagnoses and comorbidities.

Still, it is heartening that when both transferring and receiving hospitals participated in HIEs, mortality was significantly lower, even after adjusting for diagnostic discordance. That these HIEs may have saved lives is encouraging: a main goal of the billions of dollars invested in EHRs through the Health Information Technology for Economic and Clinical Health (HITECH) Act, enacted as part of the American Recovery and Reinvestment Act (ARRA) in 2009, ${ }^{16}$ was to allow patients' data to follow them wherever they seek care. Yet only $3.9 \%$ of the patients in the study by Usher et al. were cared for in hospitals where both reported being part of an HIE. These early HIE adopting hospitals are highly likely to be different in other important ways, such as being actively involved in teaching or research or having enlightened leaders willing to share patient data with other, perhaps competing, hospitals and health systems.

Though far from conclusive, the study by Usher and colleagues adds important information to the body of evidence supporting the adoption and use of HIEs to share information among systems sharing responsibility for the same patients. As technologies such as FHIR $^{17}$ and CommonWell ${ }^{18}$ make realtime sharing of EHR data between health care providers more available at lower cost, it behooves us to study their effects in increasingly rigorous ways, for example, randomizing the order in which such technologies are rolled out to clinical venues and using a step-wedge design to assess their effects. ${ }^{19}$ Only then can we understand the return on our increasingly expensive investments in digital health tools and find effective ways to wield them to enhance the safety and outcomes of the care we provide to the patients we serve.

Corresponding Author: William M. Tierney, MD; Department of Population Health, Dell Medical School, University of Texas at Austin, Austin, TX, USA (e-mail: tierney@utexas.edu).

\section{REFERENCES}

1. McDonald CJ, Murray R, Jeris D, Seeger J, Blevins L. A computerbased record and clinical monitoring system for ambulatory care. Am J Public Health 1977; 67:240-5

2. McDonald CJ, Blevens L, Tierney WM, Martin DK. The Regenstrief Medical Records. MD Comput 1988; 5:34-47.

3. McDonald CJ, Tierney WM. The Medical Gopher - A microcomputer system to help find, organize and decide about patient data. West J Med 1986; 145:823-829.

4. Tierney WM, Overhage JM, McDonald CJ, Wolinsky FD. Medical student and housestaff opinions of computerized order-writing. Acad Med 1994; 69:386-89.

5. McDonald CJ, Hui SL, Smith DM, et al. Reminders to physicians from an introspective computer medical record. A two-year randomized trial. Ann Intern Med 1984; 100:130-38.

6. Overhage JM, Tierney WM, McDonald CJ. Design and implementation of the Indianapolis Network for Patient Care and Research. Bull Med Libr Assoc 1995; 83:48-56.

7. Biondich PG, Grannis SJ. The Indiana Network for Patient Care: An integrated clinical information system informed by over thirty years of experience. J Public Health Manag Pract 2004; Suppl 1:S81-6.

8. The Indiana Health Information Exchange. Available at: http://www.ihie. org. Accessed May 21, 2018.

9. Rudin RS, Motala A, Goldzweig CL, Shekelle PG. Usage and effect of health information exchange: A systematic Review. Ann Intern Med 2014; 161:803-11.

10. Hersh WR, Totten AM, Eden KB. Outcomes From Health Information Exchange: Systematic Review and Future Research Needs. JMIR Med Inform 2015; 3:e39.

11. Rahurkar S, Vest JR, Menachemi N. Despite the spread of health information exchange, there is little evidence of its impact on cost, use, and quality of care. Health Aff (Millwood) 2015; 34:477-83.

12. Menachemi N, Rahurkar S, Harle CA, Vest JR. The benefits of health information exchange: An updated systematic review. J Am Med Inform Assoc 2018. https://doi.org/10.1093/jamia/ocy035.

13. Usher M, Sahyni \&, Herrigel D, et al. Diagnostic discordance, health information exchange and inter-hospital transfer outcomes: a population study. J Gen Intern Med 2018; https://doi.org/10.1007/s11606-0184491-x.

14. Agency for Healthcare Research and Quality. Healthcare Cost and Utilization Project. Available at: https://www.ahrq.gov/research/data/ hcup/index.html. Accessed May 21, 2018.

15. Riesenberg LA, Leitzsch J, Massucci JL, Jaeger J, Rosenfeld JC, Patow C, et al. Residents' and Attending Physicians' Handoffs: A Systematic Review of the Literature. Acad Med. 2009; 84:1775-178.

16. H.R.1 - American Recovery and Reinvestment Act of 2009. Available at: https://www.congress.gov/bill/111th-congress/house-bill/1. Accessed May 22, 2018.

17. Bender D, Sartipi K. HL7 FHIR: An Agile and RESTful approach to healthcare information exchange. Proceedings of the 26th IEEE International Symposium on Computer-Based Medical Systems, Porto, 2013:326-31.

18. Commonwell Health Alliance. Available at: http://www. commonwellalliance.org/. Accessed May 21, 2018.

19. Celia A Brown, Richard J Lilford. The stepped wedge trial design: A systematic review. BMC Med Res Methodol 2006; 6: 54. 